\title{
Boson Mediated Relaxation in Solids
}

\author{
Douglas H. McQueen and J. Kubát \\ Department of Polymeric Materials, Chalmers University of \\ Technology, S-41296 Göteborg, Sweden
}

\begin{abstract}
Dielectric and mechanical relaxation in solids, characterised by non-exponential, extended relaxation spectra, is analysed taking the interaction of the phonon (boson) field with the relaxing elements in the solid explicitly into account. Combination of Einstein's rate equations with Bothe's analysis of boson clustering results in a second order non-linear rate equation for the relaxing elements. The character of the solutions of this equation is governed by the strength and frequency dependence of the interaction of the phonons with the relaxing elements. The upper frequency limit is the Debye cutoff frequency while the lower frequency limit is determined by grain boundaries or the like. Relaxations extending over four decades of time are obtained using physically realistic parameters. Fourier transformation produces a $1 / \mathrm{f}$ spectrum over a correspondingly wide frequency range. These results are compatible with experimental results concerning stress relaxation in solids and with the "universal dielectric response" of dipoles in condensed matter.
\end{abstract}

\section{INTRODUCTION}

Some important phenomena of classical physics, such as dielectric and mechanical relaxation and electrical $1 / \mathrm{f}$ noise, are characterised by extensive interactions between the elements taking part in the processes and by non-linear effects. These characteristics can often be modelled using relatively simple mathematical expressions in which some of the terms can be given physical meanings. However, many of the mathematical models used (relaxation time spectra, stress dependent thermal activation, power laws, Williams-Watts functions) are either empirical or based on assumptions and ideas not necessarily well founded in basic physics. The tests of the validities of the models are almost always comparisons with experimental results, and although they have extensive similarities, different materials show slightly different characteristics (Kubát 1965, Ek et al. 1987) and the result is a number of competing models of the phenomena with little indication of which model should be applicable to what material. Further, the physical interpretation of the various parameters obtained through fitting the mathematical expressions to the experimental data is difficult to impossible in many cases. Clearly, what one would 
like is a model based on fundamental physics and chemistry and containing coefficients that can be determined independently by experiment or calculation.

There has been considerable progress in this direction in recent years, examples of which are theories of low energy correlated states, especially as developed by Ngai $(1979,1980)$, and of fractal structures, for instance as developed by Niklasson (1987). Still, discrepancies between theoretical predictions and experimental results are frequency found (Niklasson 1987), indicating that while the theories are generally correct their predictions are not easily tailored to particular cases.

One of the roots of work in this area concerns transitions of particles between two energy levels separated by a potential barrier. An example of early work on this problem is that of Kramers (1940) concerning a diffusion model of chemical reactions. The particles surmount the potential barrier assisted by Brownian motion, the energy for which is supplied by the thermal bath. Kramers showed that in most cases the results of his calculations are essentially equivalent to the results of the "transition state method" used in the analysis of chemical reactions. Kramers' classical model does not concern tunneling effects.

More recently there has been considerable work on low temperature systems in which the transitions between the two energy states are affected by quantum mechanical tunneling (Reichman and Silbey 1995, 1996). The relaxing two state system is coupled to a bath of quantum mechanical harmonic oscillators. To calculate correctly the dynamics of the system, the states of the density matrix have to include the phonon bath as well as the system itself. This work as well as that of others in the field is formulated in quantum dynamical terms that do not easily lend themselves to interpreting for instance stress relaxation experiments carried out at room temperature. In the following we therefore restrict ourselves to classical theory and applications to relaxing systems at "ordinary" temperatures.

Stress relaxation is an especially simple instance of a relaxation phenomenon where the similarities between various materials such as metals and polymers are clearly evident. It has been found that the inflection slope $\mathrm{F}$ of the stress vs. In time curves is about $10 \%$ of the total relaxed stress. This means that when approximated by straight lines the curves extend over about four decades of time (Kubát 1965). Figure 1 shows examples (lead, polyisobutylene, cadmium and polyethylene in tension at room temperature) redrawn from the work of Kubát (1965). This empirical finding was one of the earliest examples of so-called universal behaviour. It was later corroborated in a review focused on the behaviour of polymers (Kubát and Rigdahl 1986).

A model based on the time domain equivalent of the co-operative mechanism underlying BoseEinstein statistics which emulates the kinetics of the stress relaxation process (Högfors et al. 1981, Kubát et al. 1992) has been formulated. A particular feature of this model is the appearance of clusters of elementary transitions as required by the type of statistics used. This results in relaxation time spectra in qualitative agreement with experimental evidence.

The present contribution is an attempt to take a step from the largely phenomenological description described above to a quantitative model in which the interaction of the phonons with the relaxing entities is taken explicitly into account. 


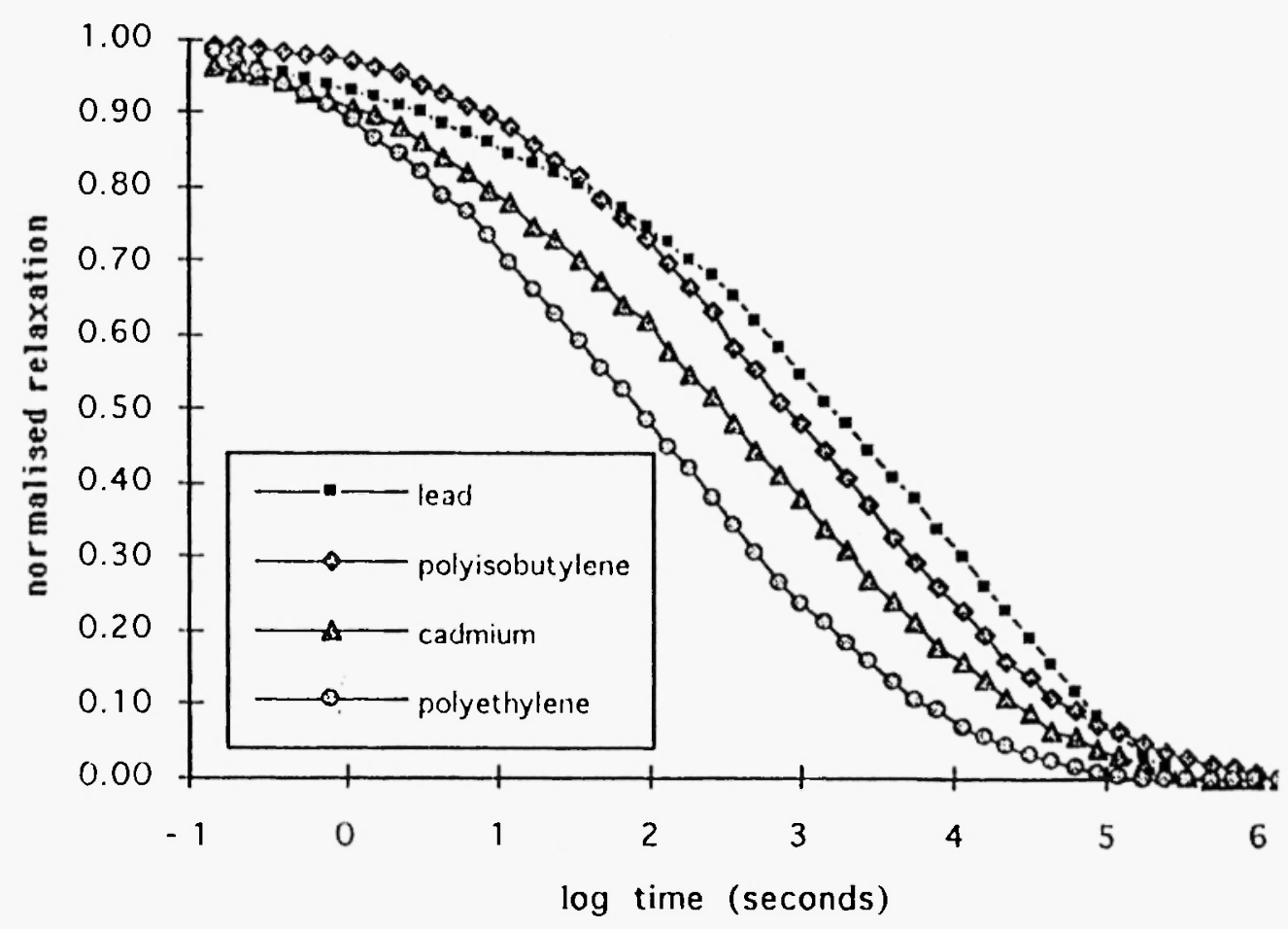

Fig. 1: Relaxation plots redrawn from Kubát (1965) for lead, polyisobutylene, cadmium and polyethylene in tension at room temperature. The relaxation extends over about four decades in time.

In qualitative terms the model we propose is the following. When a solid body is stretched (or otherwise stressed) by an external force, some elements of the body (defects, dislocations or the like) are pushed over some potential barrier into an upper energy level, overpopulating that level. The process is seen as reversible and the overpopulation slowly disappears (relaxes) through spontaneous transitions from the upper energy level to the lower one and stimulated transitions between the two levels. The result is creep or stress relaxation.

If there were only spontaneous transitions from the upper level to the lower one the relaxation would be exponential with a single (long) time constant. However, in the present approach stimulated transitions involving clusters of low energy phonons are included in the process. We find that in solids this leads to a spectrum of successively shorter time constants resulting in a relaxation that extends over typically about four orders of magnitude in time.

The process is one of induction in the following way. When a cluster of phonons, say a triplet, stimulates a transition from the upper energy level to the lower one the cluster gains a phonon. This quadruplet has a somewhat shorter lifetime than the triplet, implying that the next transition happens 
sooner than it would otherwise have done. On the other hand, when the triplet stimulates a transition from the lower energy level to the upper one the exiting phonon cluster is a doublet with a somewhat longer lifetime. The net result is a somewhat accelerated relaxation process. [We note that stimulated emission of phonons has been observed in ruby (Hu 1980) and in Tetrasil glass (Prieur et al. 1996).] Altogether the phonon transitions tend to be grouped or clustered in time. Obviously this also means that the transitions of the relaxing elements of the sample are also clustered in time.

In the following a non-linear differential equation for the relaxation process for a single energy level difference and upper energy level lifetime will be derived (section 3) after having established starting points in Bose-Einstein statistics (Einstein 1917) and introducing the boson clustering phenomenon following Bothe (1924) (section 2). The non-linear differential equation is then solved analytically (section 4). The size of the important quantity here denoted $B$ that determines the rate of relaxation in terms of the Einstein A and B coefficients is then estimated in terms of phonon/element interaction cross sections (section 5 ). Next the total relaxation process for a spectrum of energy level differences is constructed by combining the individual relaxations, one for each energy level difference (section 6). This produces relaxation processes similar to those observed experimentally. The amplitude of the relaxation curve is linearly proportional to the applied stress energy while its shape is independent of the applied stress, as it should be. The result is compared to the semi-empirical approach to BoseEinstein similar relaxation kinetics as developed by Kubát and co-workers (section 7) and shown to give essentially identical results. Our result is also shown to have similarities to an alternative formulation by Ngai and co-workers (1987) which describes a "universal form of relaxation" (Jonscher 1977) (section 8).

\section{STARTING POINTS}

The roots of the following calculations are contained in the basic formulae of Bose-Einstein statistics. Consider a system in which there are two energy levels with energies $\varepsilon_{1}$ and $\varepsilon_{2}$ corresponding to an energy difference $h v=\varepsilon_{2}-\varepsilon_{1}$ such that the numbers of elements $N_{1}$ and $N_{2}$ in the respective energy levels are

$$
\begin{aligned}
N_{1} & =p_{1} \exp \left(-\varepsilon_{1} / k T\right) \\
\text { and } & \\
N_{2} & =p_{2} \exp \left(-\varepsilon_{2} / k T\right)
\end{aligned}
$$

according to Boltzmann, where $\mathrm{p}_{1}$ and $\mathrm{p}_{2}$ are the statistical weights (degeneracies) of the respective energy levels. The elements can exchange phonons and thereby move from one energy level to the other. Then according to Einstein (1917) one has 


$$
\frac{\mathrm{dT}_{\mathrm{UL}}}{\mathrm{at}}=\mathrm{a}_{1} \mathrm{~N}_{2}+\mathrm{b}_{2} \mathrm{~N}_{2} \mathrm{w}(\mathrm{v})
$$

and

$$
\frac{\mathrm{dT} T U}{\mathrm{dt}}=b_{1} N_{!} w(v)
$$

where $w(v)$ is the energy density per unit frequency in the boson field and where $T_{U L}$ designates a transition from the upper energy level (2) to the lower one (1) by emission of a boson, and $\mathrm{T}_{\mathrm{LU}}$ denotes a transition from the lower energy level (1) to the upper one (2) accompanied by the absorption of a boson. The radiation powers exchanged in such transitions are found by multiplying $\mathrm{dT}_{\mathrm{UL}} / \mathrm{dt}$ and $\mathrm{dT}_{\mathrm{LU}} / \mathrm{dt}$ by $\mathrm{hv}$. At equilibrium, the two powers must be equal, and one finds:

$$
w(v)=\frac{a_{1} / b_{2}}{\frac{b_{1} p_{1}}{b_{2} p_{2}} \exp \left[\left(\varepsilon_{2}-\varepsilon_{1}\right) / k T\right]-1}
$$

which is of the same form as the result obtained by multiplying the density of phonon states in three dimensions, $\mathrm{D}(v)=2 v^{2} / \mathrm{v}^{3}$, ( $v$ being the group velocity of the phonons) by the average quantum occupation number of a given state $<n>=1 /[\exp (h v / k T)-1]$, when $a_{1} / b_{2}$ is identified with $2 v^{2} / v^{3}$, since Einstein showed that $b_{2} p_{2}=b_{1} p_{1}$. The density of states effectively cuts off at the Debye frequency $v_{D}$, which can be taken into account by multiplying $D(v)$ by $\exp \left(-v / \nu_{D}\right)$, as suggested by Roland and Ngai (1997). This formula for $w(v)$ is obtained only when induced emission is present, in addition to spontaneous emission and absorption.

Following Bothe (1924), let the phonons interacting with the solid be singlets, the volume density of which is denoted $n_{1}$, doublets, where the phonon density is $n_{2}$, triplets with corresponding density $n_{3}$, etc. Denoting by $\mathrm{dT}_{1} / \mathrm{dt}$ the rate of transitions of order $\mathrm{i}$ (for singlet, double, triplet, etc.) we find that per unit frequency

$$
\frac{d T_{1}}{d t}=N_{2} a_{1}+N_{1} b_{1} h v n_{2}-N_{2} b_{2} h v n_{1}-N_{1} b_{1} h v n_{1}
$$

which is zero at thermodynamic equilibrium. Further, we find that

$$
\begin{aligned}
& \frac{d T_{2}}{d t}=N_{2} b_{2} h v n_{1}+N_{1} b_{1} h v n_{3}-N_{2} b_{2} h v n_{2}-N_{1} b_{1} h v n_{2}, \\
& \frac{d T_{3}}{d t}=N_{2} b_{2} h v n_{2}+N_{1} b_{1} h v n_{4}-N_{2} b_{2} h v n_{3}-N_{1} b_{1} h v n_{3}, \\
& \frac{d T_{4}}{d t}=N_{2} b_{2} h v n_{3}+i_{1} v_{1} \text { ilinin }_{5}-N_{2} b_{2} h v n_{4}-N_{1} b_{1} h v n_{4},
\end{aligned}
$$

etc. 
Addition of the first s equations above gives

$$
\sum_{i=1}^{s} \frac{d T_{i}}{d t}=N_{2} a_{1}-N_{1} b_{1} h v n_{1}-N_{1} b_{1} h v n_{s}+N_{1} b_{1} h v n_{s+1}
$$

Since the total number of bosons is finite $n_{s}$ must vanish for sufficiently large $s$ and one has

$$
\sum_{i=1}^{s} \frac{d T_{i}}{d t}=v_{2^{\bar{x}}}-N_{1} b_{1} h v n_{1}=\frac{d T_{t n t}}{d t}
$$

which equation is central to our considerations of the dynamics of the fluctuations in the numbers of bosons and the populations $\mathrm{N}_{1}$ and $\mathrm{N}_{2}$ of the lower and upper energy levels. Bothe's arguments thus show that the boson field is composed of clusters of bosons (or phonons) up to a maximum cluster size. The average density of bosons per unit frequency in each cluster size s is given by

$$
\mathrm{n}_{\mathrm{s}}=\frac{\text { const }}{\mathrm{h}} v^{2} \exp [-\mathrm{sh} v / \mathrm{kT}]
$$

The total energy density per unit frequency $w(v)$ in this field at thermodynamic equilibrium is

$$
w(v)=\text { const } v^{3} \sum_{s=1}^{\infty} \exp [-\operatorname{sh} v / k T]=\frac{\text { const } v^{3}}{\exp [h v / k T]-1}
$$

which is the same as eq. (3) when const $=2 \mathrm{~h} / \mathrm{v}^{3}$. It is also the same as hv times the total number of bosons in the field, $n_{t o t}$, per unit frequency. Of course, at equilibrium $d T_{\text {tot }} / d_{T}$ is zero. Insertion of the equilibrium values for $N_{2} / N_{1}$ and for $n_{1}$ into eq. (6) when $d T_{\text {tot }} / d T$ is zero gives the relation $b_{1}=\left(p_{1} / p_{2}\right)$ $\left(v^{3} / 2 h v^{3}\right) a_{1}$, consistent with the previously obtained expression for $a_{1} / b_{2}$.

\section{NON-LINEAR RATE EQUATION}

In this section a non-linear differential rate equation for the relaxation is derived. It concerns only a single phonon energy hv. Later relaxations for a spectrum of phonon energies will be constructed from the solution to this rate equation.

Consider what can happen in a relaxing closed system with a constant total energy containing a constant number of relaxing elements $\mathrm{N}_{\text {tot }}=\mathrm{N}_{1}+\mathrm{N}_{2}$ which can be in either of two energy levels. These relaxing elements, typically defects and dislocations, correspond to only an extremely small fraction of the whole sample mass. They are awash in a large sea of phonons with which they can exchange 
energy. This phonon bath is characterised by a temperature $T$ which remains essentially unchanged during the process since the energy involved in the excitation process (the stress energy supplied to the sample) is minuscule compared to the total energy of the phonon bath. The phonon spectrum is assumed to be essentially continuous, which assumption may have to be modified for microscopic or mesoscopic samples. Should an element in the upper energy level emit a boson (phonon) the number of elements $N_{2}$ decreases by one and the number of elements $N_{1}$ increases by one, since the system is closed. The number of bosons (phonons) increases by one. Thus in this closed system we have $\mathrm{dT}_{\text {tot }}=$ $+d N_{1}=-d N_{2}$. Using this in the differential equation for $\mathrm{dT}_{\text {tot }} / \mathrm{dt}$ we have

$$
-\frac{d N_{2}}{d t}=N_{2} a_{1}-N_{1} b_{1} h v n_{1}
$$

per unit frequency. Differentiation with respect to time gives

$$
-\frac{d^{2} N_{2}}{d t^{2}}=\frac{d^{2}}{d t} a_{1}+\frac{d N_{2}}{d t}\left(b_{1} h v n_{1}\right)-N_{1} b_{1} h v \frac{d n_{1}}{d t}
$$

The factor $\left(b_{1} h v n_{1}\right)$ can be eliminated using the equation for $d N_{2} / d t$. A relation between $\mathrm{dn}_{1}$ and $\mathrm{dN}_{2} / \mathrm{dt}$ can be deduced by nothing that $\mathrm{dN}_{2} / \mathrm{dt}$ must be $-V o l \Delta v \mathrm{dn}_{\text {tot }} / \mathrm{dt}$ where the transition line width $\Delta v$ is taken into account explicitly and Vol is the sample volume. (As will be shown later, the transition line width can be estimated through detailed consideration of the phonon absorption/emission/ scattering process and knowledge of the numbers and sizes of absorbing/scattering elements in the sample, for instance on the basis of acoustical scattering theories.) Using the equilibrium expressions for $n_{1}$ and $n_{\text {tot }}$ one finds $d n_{1} / d t=\left(1-e^{-h v / k T}\right) d n_{10} / d t$. Then

$$
-N_{1}{ }_{1} \ldots . \cdots \frac{d n_{1}}{d t}=\frac{N_{1} b_{1} h v}{V o l \Delta v}\left[1-\exp \left(-\frac{h v}{k T}\right)\right] \frac{d N_{2}}{d t}
$$

In terms of these results eq. (10) takes the form

$$
\left.-\frac{\mathrm{d}^{2} \mathrm{~N}_{2}}{\mathrm{dt}^{2}}=\frac{\mathrm{dN} \mathrm{N}_{2}}{\mathrm{dt}}=1 \frac{\mathrm{N}_{\mathrm{tot}}}{\left[\mathrm{N}_{\mathrm{tot}}-\mathrm{N}_{2}\right.} \div \frac{\left(\mathrm{N}_{\mathrm{tot}}-\mathrm{N}_{2}\right) \mathrm{b}_{1} \mathrm{hv}}{\mathrm{a}_{1} \mathrm{Vol} \Delta v}\left(1-\exp \left(-\frac{\mathrm{hv}}{\mathrm{kT}}\right)\right)\right\}+\frac{1}{\left(\mathrm{~N}_{\mathrm{tot}}-\mathrm{N}_{2}\right)}\left(\frac{\mathrm{dN}}{\mathrm{dt}}\right)^{2}
$$

Using $\eta=N_{2} / N_{\text {tot }}$, the fraction of relaxing elements in the upper energy state, this can be put in the dimensionless form

$$
-\frac{d^{2} \eta}{d \tau^{2}}=\frac{1}{1-\eta}+i-n \frac{d \eta}{d \tau}+\frac{1}{(1-\eta)}\left(\frac{d \eta}{d \tau}\right)^{2}
$$


where $\tau=a_{1} t$ and

$$
\left.B=\left[\frac{N_{t o t}}{V o l n_{\text {tot }} \Delta v}\right]\left[\frac{b_{1} h v n_{t o t}}{a_{1}}\right]\left(1-\exp _{(}^{\prime}-\frac{h v}{k T}\right)\right)
$$

$B$ is a dimensionless variable involved in the relaxation rate. The first factor in $B$ is the ratio of the densities of the relaxing elements and the total number of bosons available in the frequency interval $\Delta v$ and the second factor is the ratio of stimulated to spontaneous transition rates. Note that when $b_{1}$ is zero (no stimulated emission) $\mathrm{B}$ becomes zero. It is easy to show that for $\mathrm{B}=0$ the solution to the differential equation is a simple exponential decay. The normalised number of unrelaxed elements $\eta$ is always between a positive initial value less than unity and its equilibrium value when the relaxation is complete.

\section{SOLUTIONS TO THE NON-LINEAR RATE EQUATION}

A solution to the non-linear rate equation can be obtained by noting that

$$
\frac{d}{d \tau}\left(\frac{d \eta / d \tau}{1-\eta}\right)=\frac{1}{1-\eta} \frac{d^{2} \eta}{d \tau^{2}}+\frac{1}{(1-\eta)^{2}}\left(\frac{d \eta}{d \tau}\right)^{2}
$$

which makes it possible to eliminate the squared derivative and then integrate the result to obtain

$$
\frac{\mathrm{d} \eta}{\mathrm{d} \tau}=-\mathrm{B} \eta(1-\eta)-1+C(1-\eta)
$$

with $\mathrm{C}$ a dimensionless constant of integration to be evaluated below. This is a (separable) differential equation for $\eta$. The required standard integral can be expressed in terms of the two solutions of $\eta^{2}-\eta$ $(\mathrm{B}+\mathrm{C}) / \mathrm{B}+(\mathrm{C}-1) / \mathrm{B}=0, \eta_{1}$ and $\eta_{2}\left(\eta_{1}>\eta_{2}\right)$. After a little algebra $\eta(\tau)$ can be expressed as

$$
\eta(\tau)=\eta_{2}+\frac{\eta_{2}-\eta_{1}}{A \exp \left(\left(\eta_{1}-\eta_{2}\right) B \tau\right)-1}
$$

with $\mathrm{A}$ another dimensionless constant of integration. The decaying solution with initial value $\eta_{0}$ between $\eta_{1}$ and $\eta_{2}$ has $A=\left(\eta_{0}-\eta_{1}\right) /\left(\eta_{0}-\eta_{2}\right)<0$ and $\eta_{1}-\eta_{2}=\left[(C-B)^{2}+d B\right]^{1 / 2} / B>0$. It is given by

$$
\eta(\tau)=\frac{\eta_{1}-\eta_{2}}{|\mathrm{~A}| \exp \left(\left(\eta_{1}-\eta_{2}\right) \mathrm{B} \tau\right)+1}+\eta_{2}
$$


The final equilibrium value of the number of elements in the upper energy state, $\eta_{2}$, is given by $\eta_{2}=$ $\left(p_{2} / p_{1}\right) e^{-x} /\left[1+\left(p_{2} / p_{1}\right) e^{-x}\right]$ where $x=h v / k T$ according to Boltzmann. This quantity can be used to evaluate the integration constant $C$ from $C=B \eta_{2}+1 /\left(1-\eta_{2}\right)$ since $v(\eta)=d \eta / d t$ is zero when $\eta=\eta_{2}$. Then the other solution, $\eta_{1}$, can be evaluated from $\eta_{1}=1+1 /\left[B\left(1-\eta_{2}\right)\right]$

When $\mathrm{B}=0$ the result above should be a simple exponential decay since there is no stimulated emission. For $B$ small compared to unity one finds that $B\left(\eta_{1}-\eta_{2}\right)=C$, the constant of integration. For small deviations from equilibrium implying small $\eta_{0}-\eta_{2}$ one also finds that $\mathbf{A}$ is large compared to unity and the expected simple exponential decay results.

Finally, it is necessary to construct how an applied force energises elements of the sample material that can later relax to their equilibrium state. More specifically, it is necessary to relate the initial value of $\eta_{0}$ to the equilibrium one $\eta_{2}$, as a function of $x$, the energy of the excited state. Two essentially equivalent candidates for such a relation are $\eta_{0}=\eta_{2}+\delta_{1}$ and $\eta_{0}=\eta_{2}\left(1+\delta_{2}\right)$ with both $\delta_{1}$ and $\delta_{2}$ constant and much less than unity. Here the sizes of $\delta_{1}$ and $d_{2}$ are proportional to the applied stress. In fact, as a first approximation they are proportional to the stress energy corresponding to the work done by the external force on the sample. These relations ensure a wide excitation spectrum the shape of which is essentially independent of the magnitudes of $\delta_{1}$ and $\delta_{2}$ as long as they are small (corresponding to relatively mild forces and small deformations of the material).

Now it only remains to estimate the value of $B$ before the relaxation $\eta(\tau)$ is completely determined for a given single value of the energy level difference characterised by $x=h v / k T$.

\section{ESTIMATIONS OF INTERACTION CROSS SECTIONS}

Before continuing with our analysis we note that non-exponential relaxation is seldom if ever observed in normal gases and only infrequently observed in normal liquids. This implies that the quantity $\mathrm{B}$ in the relaxation must be small compared to unity in gases and liquids. On the other hand, non-exponential relaxations are characteristic of solids in which case B must be significantly larger than unity. In this section we discuss phonon propagation in gases, liquids and solids in order to check on the expected importance of $B$ in the three cases.

The first step in estimating the value of $B$ is to substitute expressions for $b_{1} / a_{1}$ and for $n_{\text {tot }}$ giving

$$
\left.\mathrm{B}=\frac{\mathrm{N}_{\mathrm{tot}}}{\mathrm{V}_{\mathrm{ol}}} \frac{\mathrm{p}_{1}}{\mathrm{p}_{2}} \frac{\mathrm{v}^{3}}{2 v^{2} \Delta v} 1-\mathrm{e}^{-\mathrm{x}}\right)
$$

The line width of the transition between the two states, $\Delta v$, can be estimated using ordinary phonon scattering theory. The density of interacting elements $\mathrm{N}_{\mathrm{tol}} / V_{0}$ times the interaction cross section $\sigma$ is the change in the number of phonons per unit distance or $\Delta v / v$. This makes $v^{2} \Delta v$ equal to $\left(\mathrm{N}_{\text {tot }} / V_{0}\right)$ 
$\sigma v^{2} v$ and the expression for $\mathrm{B}$ becomes

$$
B=\frac{p_{1}}{p_{2}} \frac{v^{2}}{2 \sigma\left(\frac{k^{\top}}{h}\right)^{2} x^{2}}\left[1-e^{-x}\right]
$$

Now the problem of evaluating B is mainly focused on evaluation of the interaction cross section $\sigma$.

For gases and liquids "classical" sound absorption is due primarily to viscous and thermal losses. The absorption constant $\alpha$ is then proportional to the square of the frequency and can be written $\alpha=\alpha_{\mathrm{f}} \mathrm{f}^{2}$. For gases at ordinary temperature and pressure in the MHz region $\alpha_{\mathrm{f}}$ is around $10^{-11} \mathrm{~s}^{2} / \mathrm{m}$ (Kuttruff 1991). Extrapolation of this attenuation shows that no propagating acoustic waves can exist for frequencies above about $300 \mathrm{MHz}$. Thus phonons with energies within a few orders of magnitude of $\mathrm{kT}$ (corresponding to about $6 \mathrm{THz}$ at room temperature) do not propagate and thus cannot lead to stimulated transitions in gases.

For "classical" liquids $\alpha_{\mathrm{f}}$ is around $10^{-14} \mathrm{~s}^{2} / \mathrm{m}$ (Kuttruff 1991) and extrapolation shows that no propagating acoustic waves can exist above frequencies around $70 \mathrm{GHz}$. However, at these high frequencies the viscosity is "frozen out" and damping is correspondingly reduced. Wave propagation and damping, due partly to thermal effects, take on new forms at $\mathrm{GHz}$ and $\mathrm{THz}$ frequencies. Further, in most liquids the speed of sound, which enters quadratically into the expression for $B$, is about a third that in typical solids. Thus it seems possible, but not necessarily probable, that propagating acoustic waves can affect relaxation in normal liquids.

For solids Rayleigh scattering (proportional to the fourth power of the frequency) from grain boundaries and the like is generally dominant in the $\mathrm{MHz}$ frequency region (Papadakis 1965). In the high $\mathrm{MHz}$ region stochastic scattering (proportional to the square of the frequency, as is "classical" damping in gases and liquids) takes over. In the middle and high $\mathrm{GHz}$ region and low $\mathrm{THz}$ region Brillouin scattering experiments and Raman scattering experiments show that there exist propagating phonons with damping such that the ratio $\beta$ of the line width of the propagating mode to the frequency shift is typically between about 0.1 and 0.01 . Thus $\alpha_{x}$, the damping per unit length, is $\beta k=\beta 2 \pi \mathrm{v} / \mathrm{v}$ where $k$ is the wavenumber. This attenuation coefficient is essentially the cross section, $\xi 2 \pi \mathrm{a}^{2}$, with $\xi$ a dimensionless coefficient usually between about 0.01 and 0.1 , times the density of absorbers, $\mathrm{N}_{\mathrm{tor}} / \mathrm{Vol}=$ $1 / d^{3}$, where $d$ is the centre to centre distance between absorbers. Using $d=\eta$ a with $\eta>2$ gives $a=$ $\xi v / \beta \eta^{3} v$, that is, an effective interaction radius equal to $\xi / \beta \eta^{3} \approx 1 / \eta^{3}$ times the wavelength $v / v$. This gives $\sigma v^{2}=2 \pi v^{2} \xi^{3} / \eta^{6} \beta^{2}$ in the denominator of $\mathrm{B}$ and for $\mathrm{B}$ itself

$$
\mathrm{B}=\frac{\mathrm{p}_{1}}{\mathrm{p}_{2}} \frac{\left(1-\mathrm{x}^{-\mathrm{x}}\right)}{4 \pi}=\frac{\mathrm{p}_{1}}{\mathrm{p}_{2}} \frac{\left(1-\mathrm{e}^{-\mathrm{x}}\right)}{2(2 \pi)^{2}} \frac{1}{\xi(\mathrm{ka})^{2}}
$$


using $2 \pi \xi / \eta^{3} \beta=$ ka relating $\xi, \eta$ and $\beta$. In the present model it seems appropriate that the phonons interact with the relaxing elements of the material at volumes or surfaces small compared to the wavelength, that is, ka should be small compared to unity. With $\beta$ and $\xi$ small fractions of unity and of about the same order of magnitude and $\eta$ probably around 10 or more this ka small criterion is satisfied. Inserting reasonable values of the parameters involved $\left(\mathrm{p}_{1} / \mathrm{p}_{2}=1, x=0.1, \xi=0.1, \beta=0.05\right.$ and $\eta=8$ ) we find $B \approx 5000$, significantly larger than unity.

We do not insist that the above treatment is the only way to estimate the value of the important parameter B. It seems to us to be a fairly good first approximation because the relaxation energies we deal with are small, of the order of $\mathrm{kT}$ or less, even much less, implying that the sizes of the defects should be relatively small. Phonon scattering from defects small compared to the phonon wavelength is largely independent of their shape, which makes the analysis easier than it would be for large or extended defects. Thus the results of our estimations above are relatively insensitive to the exact details of the defects and therefore relatively insensitive to the specific material under study. This is in keeping with the observation that stress relaxation in a large variety of materials can be described by the same inflection slope $\mathrm{F}$ of the stress vs $1 \mathrm{n}$ time curves, about 0.10 times the total relaxed stress (Kubát 1965).

We conclude that phonon (boson) induced relaxation transitions should normally be important in solids, insignificant in liquids and non-existent in gases. It is appropriate to continue our analysis focusing only on solids.

\section{RELAXATION CURVES}

The total relaxation of the sample must be a sum of the relaxations for each phonon energy hv as given by the solution to the non-linear differential equation. The number of relaxations as a function of $h v$ is the product of the density of the phonons per frequency interval $D(v)<n>$ and the probability that these phonons interact with an element that can relax, which is proportional to the cross section $\sigma(v)=$ $\left(2 \pi v^{2} / v^{2}\right)\left(\xi^{3} / \beta^{2} \eta^{6}\right)$. This can be written $r(v) \eta(\tau)$ where $\eta(\tau)$ is the relaxation of the interacting elements and $r(v)$ is the weighting factor in terms of the energy $h v$ :

$$
r(v)=\frac{4 \pi}{v} \frac{\varepsilon^{3}}{\beta^{2} \eta^{6}} \frac{e^{-v / v_{0}}}{e^{\text {hv/kT }}-1}
$$

Thus the total relaxation process is given by the integral

$$
\eta_{T}(\tau)=\int_{0}^{x} r(v) \eta(v) d v
$$


where the subscript $\mathrm{T}$ serves to emphasise that now all different phonon frequencies are taken into account.

When $h v$ exceeds $k T$ the exponential factor $1 /[\exp (h v / k T)-1]$ cuts the distribution off. When the Debye frequency corresponds to a temperature near $\mathrm{kT}$ or lower it also cuts the distribution off. This means that the weighting factor makes low $\mathrm{THz}$ phonons most interesting, middle and high $\mathrm{THz}$ phonons being excluded by the thermal and Debye cut-offs.

When $h v$ is small compared to $\mathrm{kT}$ the distribution $\mathrm{r}(v)$ is inversely proportional to the energy or frequency, which situation cannot extend to arbitrarily low frequencies (infrared divergence). On the other hand, while liquids are usually essentially homogeneous throughout their volumes, solids are characterised by high energy internal boundaries, for instance grain boundaries. In metals the characteristic diameter of such grains is often of the order of 50 microns. Then phonons with mean free paths greater than this characteristic distance should not be very important for the relaxation as their lifetimes are determined by collisions with grain boundaries or the equivalent or even the boundaries of the sample (often only millimetres thick). This puts a lower (soft) bound on the phonon frequency spectrum defined by $v / 2 \pi \beta \mathrm{I}_{\mathrm{ch}}$ with $\mathrm{I}_{\mathrm{ch}}$ being the characteristic length for the material at hand.

This lower bound should not take the form of a sharp cut-off but should rather be gradual, reflecting a distribution of characteristic lengths (for instance grain sizes) in the material as well as the softness of the restriction above. In the following the window function $w(x)=\left[\tan ^{-1}\left(x_{\max } / x\right)-\tan ^{-1}\left(x_{\min } / x\right)\right]$ is used with $x_{\max }$ set to 1000 so as not to cut off the distribution at high frequencies. With $l_{c h}=50$ microns $x_{1 \text { inin }}$ is $5.1 \times 10^{-5}$ allowing for more than four decades of frequency or energy in the spectrum. Somewhat smaller values of $l_{c h}$ can be used as well as much larger values. Finally the relaxation can be expressed as

$$
\begin{aligned}
& \eta_{\mathrm{T}}(\tau)=\int_{0}^{\infty}\left(\tan ^{-1}\left(v_{\max } / v\right)-\tan ^{-1}\left(v_{\min } / v\right)\right) \times \\
& \left(\frac{4 \pi}{\mathrm{v}} \frac{\xi^{2}}{\beta^{2} \eta^{6}} \frac{\mathrm{e}^{-v / v_{o}}}{\mathrm{e}^{\mathrm{hv} / \mathrm{kT}}-1}\right)\left(\frac{\eta_{1}-\eta_{2}}{\mid \mathrm{A} / \exp \left(\left(\eta_{1}-\eta_{2}\right) \mathrm{B} \tau\right)+1}+\eta_{2}\right) \mathrm{d} v
\end{aligned}
$$

where $\mathrm{v}_{\max }$ and $\mathrm{v}_{\min }$ correspond to $\mathrm{kTx_{ \operatorname {max } }} / \mathrm{h}$ and $\mathrm{kTx_{ \operatorname {min } }} / \mathrm{h}$. We have assumed that the distribution of the pairwise energy differences corresponding to the frequencies $v$ is essentially even throughout the range of energies of interest here, from near the lower cut-off frequency to near the Debye frequency $v_{D}$. It may in fact be a function of the frequency, for instance with a maximum at some intermediate frequency (Reichman and Silbey 1995, 1996), in which case the window function above can be taken as partly reflecting this situation.

Relaxation curves based on the form $\eta_{0}=\eta_{2}+\delta_{1}$ and the above expression are shown in Figure 2. For the middle curve we have used our best estimates of "typical" values of the parameters entering into the calculation: $T=300 \mathrm{~K}, \mathrm{p}_{\mathrm{J}} / \mathrm{p}_{2}=1, \xi=0.1, \mathrm{v}=5000 \mathrm{~m} / \mathrm{s}, \beta=0.05, \eta=8, \mathrm{x}_{\mathrm{D}}=1, \mathrm{l}_{\mathrm{ch}}=100$ microns, $x_{\max }=1000$ and $\delta_{1}=0.01$. The maximum normalised slope of the relaxation curve is near 


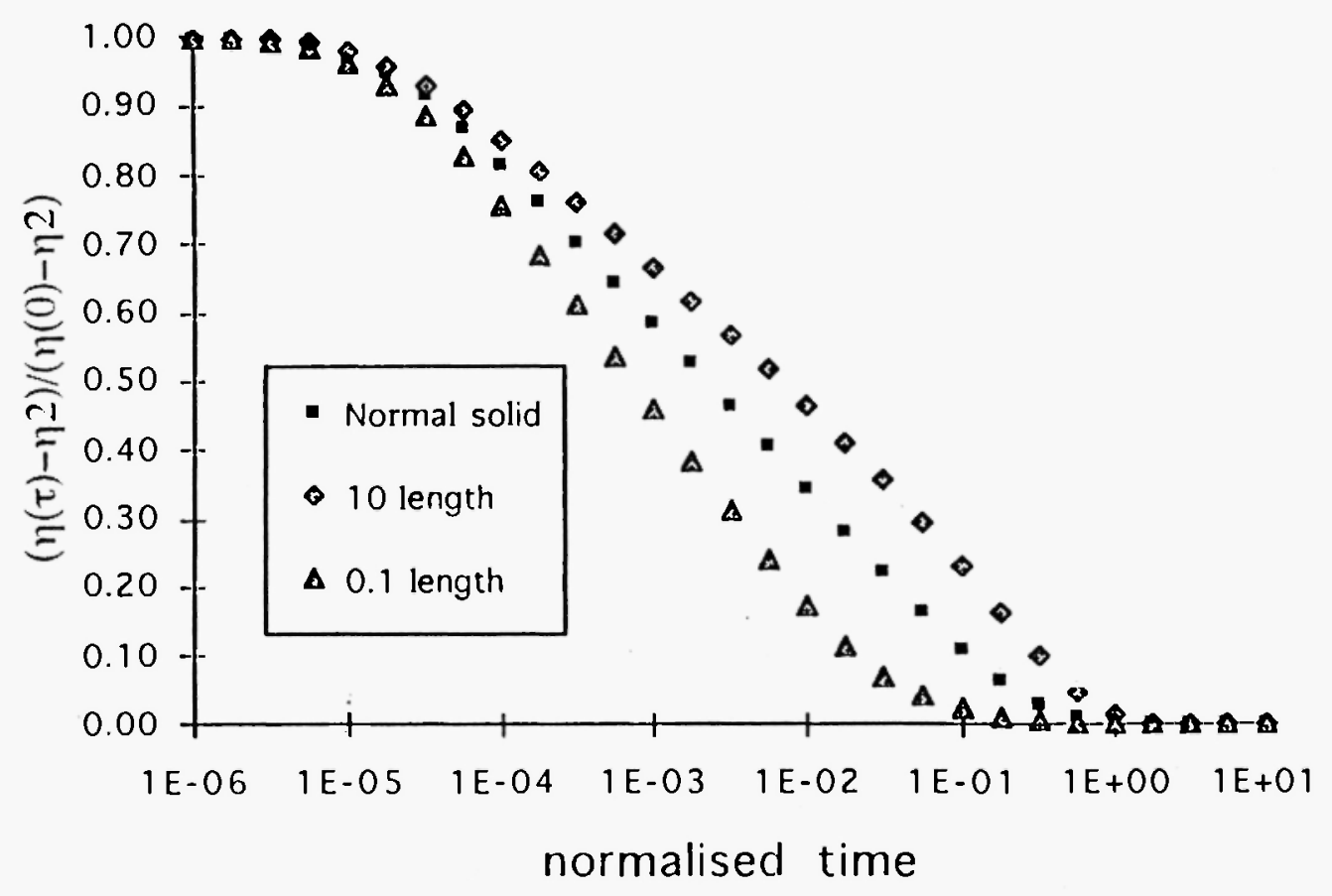

Fig. 2: Relaxation plots according to eq. (24) using the following data for the middle curve: $\mathrm{T}=300$ $\mathrm{K}, \mathrm{p}_{\mathrm{l}} / \mathrm{p}_{2}=1, \xi=0.1, \mathrm{v}=5000 \mathrm{~m} / \mathrm{s}, \beta=0.05, \eta=8, \mathrm{x}_{\mathrm{D}}=1, \mathrm{l}_{\mathrm{ch}}=100$ microns, $\mathrm{x}_{\max }=1000$ and $\delta_{1}=0.01$. The other two curves were constructed using the same values but with the material characteristic length $l_{\mathrm{ch}}$ of the upper curve equal to a millimetre and that of the lower curve equal to ten microns.

-0.1 , in good agreement with empirical results on mechanical relaxation (Kubát 1965). Using the above values it is easy to confirm that $\mathrm{ka}$ is about 0.004 , much less than unity, as required. The curves in Figure 2 have shapes similar to those of the experimental curves shown in Figure 1.

The strength of the initial perturbation in terms of the applied stress energy, $\delta_{1}$, should not affect the shape of the relaxation curves as long as its size is moderate. This has been checked. The shapes of the curves are not changed when $\delta_{1}$ is increased moderately, say to 0.03 or 0.05 or decreased toward zero. In this sense the system is linear.

For the middle curve in Figure 2 the relaxation $\eta(t)$ varies from 12.905 arbitrary units initially to 12.644 units finally, that is, the change is just over $2 \%$. The plot was constructed by subtracting 12.644 arbitrary units from each data point and normalising the result so that $\eta(t)$ is unity initially and zero finally. It is easy to see why the amplitude of the relaxation is just over $2 \%$ for the chosen values. The equilibrium value of $\eta(t)$ is just under 0.5 , as can be confirmed using the expression for $\eta_{2}$ following eq. (18) with $\mathrm{p}_{1} / \mathrm{p}_{2}=1$ and $\mathrm{x}$ small. The excitation chosen, $\delta_{1}=0.01$, is thus just over $2 \%$ of the final equilibrium value. Larger values of $\delta_{1}$ produce correspondingly larger relaxation amplitudes. The 
relation is linear, as it should be.

It is obviously important to study how sensitive the form of the relaxation curve is to variations in the sizes of the parameters used in the calculation. It turns out that the shape and maximum normalised slope value of the relaxation plot are maintained for wide (but still physically reasonable) variations in $T, p_{1} / p_{2}, \xi, v, \beta, \eta, x_{D}, x_{\max }$, and $\delta_{1}$. However, the characteristic length $1_{c h}$ of the material cannot be varied as much without changing the curves. For instance, the lower and upper curves in Figure 2 show the effect of changing the characteristic length to ten microns (for instance, smaller grains), and to one millimetre (for instance, larger grains) respectively. Interestingly, the characteristic maximum value of $-\mathrm{d} \eta(\mathrm{t}) / \mathrm{d} 1 \mathrm{nt} \approx 0.1(\eta(0)-\eta(\infty))$ is still a good overall approximation in this latter case.

\section{ANALYTICAL APPROXIMATIONS AND TRANSFORMATIONS}

Kubát and co-workers (Kubát et al. 1992, Bengtsson et al. 1991) have studied a rate equation of the form

$$
\frac{d \dot{n}}{d t}=a \dot{n}+b \dot{n}^{2}=a \dot{n}\left(1+\frac{b}{a} \dot{n}\right), a<0, b>0
$$

where $\dot{\mathrm{n}}=\mathrm{dn}(\mathrm{t}) / \mathrm{dt}$ is the rate at which unrelaxed elements relax. This equation has been given a Bose-Einstein similar interpretation because of the induction term (b/a) $\dot{n}$. The differential equation can be integrated once to give

$$
\dot{\mathrm{n}}=\frac{\mathrm{a}}{\mathrm{b}} \frac{1}{\mathrm{~A} \exp (-\mathrm{at})-1}
$$

where $\mathrm{A}=1+\left(\mathrm{a} / \mathrm{b} \dot{\mathrm{n}}_{0}\right)$ with $\dot{\mathrm{n}}_{\mathrm{o}}=\mathrm{dn}(\mathrm{t}) / \mathrm{dt}(\mathrm{t}=0)$. For cases of interest $\mathrm{A}$ is $1+\varepsilon$ with $\varepsilon$ of the order of $+10^{-5}$.

A second integration gives

$$
\begin{aligned}
n(t)=n_{0} & -\frac{1}{b} \ln \left(1+\frac{b \dot{n}_{0}}{a}(1-\exp (a t))\right) \\
= & n_{f_{1}}\left[1-\frac{1}{\ln \left(1+t_{1} / t_{0}\right)} \ln \left(1+\frac{t_{1}}{t_{0}}\left(1-\exp \left(-t / t_{1}\right)\right)\right)\right]
\end{aligned}
$$

where $t_{1}=-1 / a$ and $t_{0}=1 / b \dot{n}_{0}$. By choosing appropriate values of $t_{1}$ and $t_{0}\left(t_{1}-2.5 \times 10^{-5}\right.$ and $t_{0}-$ 0.22 ) an essentially perfect fit (maximum difference 0.013 around $t_{0}=5.6 \times 10^{-6}$ ) to the relaxation curve in Figure 2 can be obtained. Thus for this particular choice of parameters $\left(\beta, \eta, l_{c h}\right.$, etc.) and excitation spectrum $\left(\eta_{0}=\eta_{2}+\delta\right)$ the relaxation is very well described by the empirical formula for $n(t)$ 
above.

This empirical equation fitting the curve in Figure 2 can be used to obtain the Fourier cosine transformation of the relaxation, that is, its frequency spectrum. One approach is to note that the expression for $\dot{n}(t)$ can be expanded in a series according to

$$
\dot{n}(t)=\frac{-1}{b \tau} \sum_{s=1}^{S_{\max }}\left(A^{-s} \exp (-s t / \tau)\right)
$$

and consequently

$$
\dot{n}(t)=\frac{-1}{b \tau} \sum_{s=1}^{c} \frac{A^{-s}}{s} \exp (-s t / \tau)
$$

The Fourier cosine transform of this sum is easy to carry out term by term and one obtains

$$
\sum_{s=1}^{S_{\max }} \frac{A^{-s}}{s} \frac{1}{1+(\omega \tau / s)^{2}}
$$

When $A$ can be approximated by 1 and when $s_{\max }$ is large (both assumptions being true in cases of interest here) this series can be approximated by an integral which can be carried out to obtain

$$
\int_{\tau / s_{\max }}^{\tau} \frac{\mathrm{dx}}{1+(\omega \mathrm{x})^{2}}-\frac{1}{2 \omega}\left[\tan ^{-1}\left(\frac{\omega \tau}{s_{\max }}\right)-\tan ^{-1}(\omega \tau)\right],
$$

a result already obtained by Bittel and Storm (1971) among others. The integration shows that for frequencies $\omega$ below $1 / \tau$ the Fourier cosine transform is frequency independent, for intermediate frequencies up to $s_{\max } / \tau$ it is $1 / f$ and for the highest frequencies it is $1 / f^{2}$. The parameter $s_{\max }$ is essentially the maximum phonon cluster size. It clearly determines the width of the frequency interval in which the Fourier cosine transform has $1 / \mathrm{f}$ character.

\section{DISCUSSION}

It is instructive to divide $w(v)$, the energy density per unit frequency in the boson field, into its stationary or equilibrium part $w^{*}(v)$ and the deviation from that stationary part, $\delta w(v, t)$, and then insert this into the equation for $\mathrm{dT}_{\mathrm{UL}} / \mathrm{dt}-\mathrm{dT}_{\mathrm{LU}} / \mathrm{dt}=-\mathrm{dN}_{2} / \mathrm{dt}$. One obtains 


$$
-\frac{d N_{2}}{d t}=\left(N_{2} b_{2}-N_{1} b_{1}\right) \delta w(v, t)
$$

because by construction the terms in $w^{*}(v)$ do not contribute to $\mathrm{dN}_{2} / \mathrm{dt}$. An analogous equation for $\mathrm{dN}_{1} / \mathrm{dt}$ is easily obtained. These two equations involving the deviation from the equilibrium state, $\delta w(v, t)$, are analogous to the two equations Ngai and Rajagopal (1987) studied in detail,

$$
\frac{d p_{1}(t)}{d t}=-W(t)\left(p_{1}(t)-p_{2}(t)\right), \frac{d p_{2}(t)}{d t}=-W(t)\left(p_{2}(t)-p_{1}(t)\right)
$$

where $p_{1}(t)$ and $p_{2}(t)$ are the populations of the two levels and $w(t)$ is the time dependent jump transition rate. Thus when $b_{1}=b_{2}$ eq. (32) and eq. (33) are formally equivalent and $W(t)$ and $b_{1} \delta w(v, t)$ play equivalent roles. In the present work $\delta w(v, t)$ has not been evaluated. On the other hand, Ngai and Rajagopal made use of a Wigner random matrix Hamiltonian to describe the large heat bath in which the relaxing system is immersed. It has small energy level spacings of the order of $10^{-4} \mathrm{eV}$ or less corresponding to frequencies of the order of $10^{10} \mathrm{~Hz}$. This procedure leads to the "universal form of relaxation" given by Jonscher (1977) and by Ngai $(1979,1980)$. In terms of the time domain relaxation function it is

$$
f(t)=c_{1} t^{-n} \exp \left(\left(-c_{2} t\right)^{1-n}\right.
$$

where $0<\mathrm{n}<1$ and $0<\mathrm{c}_{2}$ (Ngai 1979). Here $\mathrm{n}$ is an empirical parameter used to characterise relaxation spectra and not easily susceptible of determination by independent means. The widest relaxations are found for the hardest solids and correspond to $\mathrm{n}$ around 0.7 and higher. Increasing the temperature shifts the loss peak to a higher frequency corresponding to a shorter relaxation time. For long times $\left(c_{2} t \gg 1\right)$ and for $n=0.72$ using eq. (34) we obtain a relaxation with maximum slope $-\mathrm{dn}(\mathrm{t}) / \mathrm{d} l \mathrm{nt}) / \mathrm{n}(0)$ equal to 0.1 . Incidentally, values of $\mathrm{n}$ around 0.72 are typical of percolating systems and fractal structures, as pointed out by Niklasson (1987). According to Niklasson, attempts to predict the exact value of $\mathbf{n}$ for a given system on the basis of fractal time processes or fractal structures have not always been successful.

It thus appears that the results derived here on the basis of Bose-Einstein relaxation and those of Jonscher and Ngai are largely equivalent for long times, the main difference being that the important parameter $\mathrm{n}$ in the Jonscher and Ngai result cannot easily be predicted accurately or determined independently while in principle the interactions of phonons with the relaxing material involved in the present model can be investigated by independent techniques. The details of the latter are also explicitly material and sample dependent but the general character of the interaction is determined by the requirement of a broad frequency spectrum with physically reasonable low and high frequency cutoffs. 
In a way analogous to the above, it is instructive to divide the number of singlet bosons $n_{1}$ into two parts, a stationary part $n_{1}^{*}(v)$ and its deviation from that stationary part, $\delta n_{1}(v, t)$. Using the expression for $\mathrm{dN}_{2} / \mathrm{dt}$ in terms of $\mathrm{n}_{1}$ one finds

$$
\frac{\mathrm{dN}_{2}}{\mathrm{dt}}=\mathrm{N}_{1} b_{1} h v \delta n_{1}(v, t)
$$

This can be combined with the previous expression for $\mathrm{dN}_{2} / \mathrm{dt}$ in terms of $\delta \mathrm{w}(\mathrm{v}, \mathrm{t})$ to obtain

$$
\delta n_{!}=\left[1-\frac{b_{2} p_{2}}{b_{1} p_{1}} \exp (-h v / k T)\right] \frac{\delta w(v, t)}{h v}=[1-\exp (-h v / k T)] \delta n_{t o t}
$$

relating the deviation in the number of singlets to the deviation in the total number of bosons. This was previously used in connection with eq. (11) using the equilibrium expressions for $n_{1}$ and $n_{\text {tol }}$. Clearly, when hv/kT is large all the relaxation is carried by singlets while when it is small it is distributed over the various multiplets. It does not seem possible to find expressions similar to the above for $\delta n_{2}, \delta n_{3}$, etc., in terms of $\delta n_{\text {tot. }}$

\section{CONCLUSION}

In the above we have shown that characteristic relaxation spectra for solids can be obtained by setting up and solving rate equations that take both the Einstein A and B coefficients into account. All the material constants entering into the result can in principle be determined independently in experiments. This is in contrast to the semi-empirical equations presently used for characterising mechanical relaxation in solids.

An important parameter in the relaxation is the quantity B which is proportional to the Einstein B coefficient and explicitly dependent on the phonon energy $h v$. When the Einstein B coefficient is ignored, as is often the case, the quantity $B$ is zero, there is no induction and the relaxation is a simple exponential decay.

Similarly, for a single phonon energy there is a single exponential decay. Only for a wide range of phonon energies corresponding to a wide range of energy level differences is there a correspondingly wide range of relaxation rates. We have been able to establish (soft) limits for this range of phonon energies by considering the Debye temperature on the one hand and the longest possible relevant phonon wavelengths on the other. These limits are rather robust in the sense that they apply to most solids regardless of their specific characteristics. This is in agreement with observations concerning the characteristic broad mechanical relaxation spectra of most solids.

The detailed shapes of mechanical relaxation spectra in solids do vary somewhat, as is especially 
well illustrated in Li plots. This is a consequence of the fact that the phonon spectra of the solids can vary somewhat as well as can the details of how the applied mechanical stress moves the relaxing elements to their excited states.

Finally, we have shown that the results obtained here are compatible with results obtained by Ngai, Jonscher and Kubát, among others.

\section{REFERENCES}

P. Bengtsson, C. Klason, D.G. Kubát, J. Kubát and D.H. McQueen. 1/f noise and Bose-Einstein-like statistics, in: Noise in Physical Systems and l/f Fluctuations, T. Musha, S. Sato and M. Yamamoto (Eds.), Japan: Ohmsha Ltd., 1991.

H. Bittel and L. Storm. Rauschen, Heidelberg: Springer-Verlag, 1971; p. 194.

W. Bothe. Zeitschrift für Physik, 20, 145-52 (1923).

A. Einstein. Physikalische Zeitschrift, 18, 121-28 (1917).

C.-G. Ek, J. Kubát and M. Rigdahl. Colloid and Polymer Sci., 265, 803-9 (1987).

P. Hu. Phys. Rev. Lett., 44, 417-20 (1980).

C. Högfors, J. Kubát and M. Rigdahl. Phys. Stat. Sol. (b), 107, 147-55 (1981).

A.K. Jonscher. Nature, 267, 673-79 (1977).

H.A. Kramers. Physica, 7, 284-304 (1940).

H. Kuttruff. Ultrasonics, London: Elsevier Applied Science, 1991; chapter VIII.

D.G. Kubát, H. Bertilsson, J. Kubát and S. Uggla. Rheol. Acta, 31, 390-98 (1992).

J. Kubát. Nature, 205, 378-79 (1965).

J. Kubát and M. Rigdahl. In: Failure of Plastics, W. Brostow and R.D. Corneliusson (Eds.), New York: Hanser, 1986; pp. 60-83.

K.L. Ngai. Comments Solid State Phys., 9, 127-40 (1979).

K.L. Ngai. Comments Solid State Phys., 9, 141-56 (1980).

K.L. Ngai and A.K. Rajagopal. A theory of non-Debye relaxation in condensed matter, in: Non-Debye Relaxation in Condensed Matter, T.V. Ramakrishnan and M. Raj Lakshmi (Eds.), Singapore: World Scientific, 1987.

G.A. Niklasson. J. Appl. Phys., 62, Rl-14 (1987).

E.P. Papadakis. J. Acoust. Soc. Am., 37, 703-10 (1965).

J.-Y. Prieur, M. Devaud, J. Joffrin, C. Barre, M. Stenger and M. Chapellier. Physica B, 219 \& 200, 235-38 (1996).

D.R. Reichman and R.J. Silbey. J. Phys. Chem., 99, $2777-81$ (1995).

D.R. Reichman and R.J. Silbey. J. Chem. Phys., 104, 1506-18 (1996).

C.M. Roland and K.L. Ngai. Mechanics of Time-Dependent Materials, 66, 109-22 (1997). 